\title{
Entrelacs
}

ENTRELACS Cinéma et audiovisuel

14 | 2018

Marchés du film : évolutions, mutations et perspectives

\section{Le marché de la coproduction de la Berlinale dans l'univers du cinéma du monde.}

\section{Ana Vinuela}

\section{OpenEdition}

\section{Journals}

Édition électronique

URL : http://journals.openedition.org/entrelacs/4332

DOI : 10.4000/entrelacs.4332

ISSN : 2261-5482

Éditeur

Éditions Téraèdre

Référence électronique

Ana Vinuela, « Le marché de la coproduction de la Berlinale dans l'univers du cinéma du monde. », Entrelacs [En ligne], 14 | 2018, mis en ligne le 18 décembre 2018, consulté le 01 mai 2019. URL : http:// journals.openedition.org/entrelacs/4332 ; DOI : 10.4000/entrelacs.4332

Ce document a été généré automatiquement le 1 mai 2019.

Tous droits réservés 


\title{
Le marché de la coproduction de la Berlinale dans l'univers du cinéma du monde.
}

\author{
Ana Vinuela
}

Les marchés de la coproduction sont des espaces de mise en relation entre producteurs de films indépendants et professionnels susceptibles de compléter leur financement. Nés à la fin du XXe siècle comme extension d'un festival ou d'un marché de films, ces événements ont connu une forte croissance depuis le début du XXIe siècle, en parallèle à la création de fonds de soutien à la coproduction et au développement d'un marché global pour le cinéma indépendant. Ils peuvent être envisagés comme des «instances de production culturelle professionnalisée [...] traversées par des idéologies et des imaginaires $»^{1}$ qui sont congruents avec ceux d'un cinéma d'auteur transnational, et promouvant un rapprochement entre les logiques éditoriales des festivals et les intérêts commerciaux des marchés du film. Si les festivals apportent aux marchés de la coproduction une légitimité culturelle et artistique, les projets en quête de financements dans le cadre de ces marchés épousent quant à eux les critères de sélection des festivals, férus de films où se matérialise le regard d'un auteur face à des sujets sociétaux ou moraux, déconnectés des formes génériques ou les réinterprétant sous des angles originaux.

Dans cet article nous nous intéresserons au marché de la coproduction de la Berlinale comme lieu d'observation de la globalisation du cinéma d'auteur et de la diversification de la notion de "cinéma du monde ». Nous allons dans un premier temps décrire le positionnement de cette manifestation au sein de la Berlinale et identifier ensuite les différents acteurs qui interviennent et leurs interactions. Enfin, nous examinerons le rôle de cet événement dans le financement et la circulation d'un cinéma d'auteur imbriqué dans l'univers du "cinéma du monde». Notre démarche intègre les apports des Film Festival Studies, qui ont mis en lumière l'hybridité et les transformations des festivals ${ }^{2}$. Nous nous intéressons en particulier à l'application dans ce cadre du modèle des parties prenantes ${ }^{3}$, pour identifier les intérêts des différents collectifs. Cette approche a été mobilisée par Marijke de Valck pour étudier le marché de la coproduction du Festival de 
Rotterdam ${ }^{4}$, mais à différence de cette auteure, qui place au centre de son analyse les contradictions et les processus de négociation entre l'art et le commerce, nous considérons le marché de la coproduction de la Berlinale comme une instance de production caractérisée par l'interpénétration de ces deux pôles. Notre objectif est de faire dialoguer une perspective socio-économique et des études sur les festivals, faisant référence à des débats actuels dans ce domaine de recherche, notamment à propos de l'expansion du périmètre des festivals et leur prise en compte des intérêts de l'industrie. Le noyau empirique de notre étude intègre des données issues des archives et des catalogues du marché de la coproduction de Berlin, ainsi que l'observation des activités de cette manifestation en 2017 et 2018, en particulier la participation à six rendez-vous entre des producteurs porteurs de projets et des éventuels partenaires. Enfin, un entretien avec Martina Bleis, sa co-directrice, nous a permis de compléter notre analyse.

\section{La place du marché de la coproduction dans la Berlinale}

3 Le marché de la coproduction de la Berlinale, à la fois une extension du festival International du Cinéma et une partie du marché du film, témoigne du processus de transformation des festivals en "cluster de créativité et de commerce $»^{5}$. Le festival de Berlin constitue la vitrine d'un cinéma politique tourné vers le réel et en prise avec des questions sociétales, qui se déroule simultanément au marché du film dans le cadre futuriste de Potsdamer Platz, symbole de la réunification des zones et des idéologies qui divisaient la ville dans le passé́. L'European Film Market (EFM), dont fait partie le marché de la coproduction, est le deuxième marché du film en Europe en nombre de participants et de transactions, après celui de Cannes. Dans le cadre du EFM, des sociétés de ventes internationales, chargées de la commercialisation des droits des films en dehors des territoires réservés aux producteurs, proposent aux acheteurs des œuvres finalisées, en développement ou en production, englobant tous les budgets, genres et nationalités, contrairement aux projets sélectionnés dans le marché de la coproduction, dont la spécificité sera présentée plus loin. En 2003, le EFM a associé le Berlinale Talents, une plateforme d'échanges favorisant le développement des carrières et la mise en réseau de jeunes professionnels du monde entier, et un an après, son périmètre s'est étendu avec l'incorporation du marché de la coproduction et du fonds de soutien à la coproduction World Cinema Fund. Ces quatre composantes intègrent la section tournée vers l'industrie, étroitement connectée au Festival.

Le marché de la coproduction a tissé des liens avec toutes les composantes de la Berlinale. D'une part, les projets de réalisateurs dont un film a été programmé dans une section du festival (ou écrit dans le cadre de la Résidence, une initiative en place entre 2012 et 2015) ont un accès privilégié au marché de la coproduction, puisque leurs producteurs sont dispensés de l'obligation d'avoir réuni au moins 30 pour cent du financement pour participer. De son côté, le festival programme chaque année des films qui ont transité par ce marché7. D'autre part, le marché de la coproduction est intégré au EFM et se déroule dans les locaux du Parlement régional, situé en face du Martin-Gropius-Bau, l'imposant bâtiment qui héberge le marché du film, ce qui permet aux professionnels de circuler facilement entre les deux espaces. Avec le World Cinema Fund, la connexion se matérialise par une dérogation accordée aux projets issus des pays en transition soutenus par ce fonds, dont le budget minimum pour accéder au marché de la coproduction s'établit à 
750000 euros au lieu d'un million exigé pour les autres projets. Enfin, le Talent Project Market, organisé en coopération avec Berlinale Talents, permet à dix jeunes producteurs de présenter leurs projets dans le marché de la coproduction et de bénéficier d'un accompagnement personnalisé pour comprendre le fonctionnement du financement international du cinéma indépendant. Ces aménagements renforcent la cohérence d'un ensemble intégré par des éléments qui possèdent des logiques autonomes, mais qui visent tous à encourager la pratique de la coproduction internationale de films, le développement d'un marché global pour le cinéma d'auteur et l'entreprenariat culturel, des objectifs qui sont également au cœur du Programme Europe Créative - MEDIA de l'Union Européenne, qui finance près de la moitié des 300000 euros que coûte l'organisation de la manifestation ${ }^{8}$.

Par ailleurs, le marché de la coproduction de Berlin fait partie d'un circuit professionnel mondial dans lequel se succèdent des manifestations qui ont des objectifs similaires, dont les plus importantes sont présentées dans le tableau 1. Les acteurs et les projets se déplacent dans ce circuit, configurant une géographie de la coproduction et contribuant à la formation d'une culture professionnelle cosmopolite et globale. Il convient néanmoins de nuancer l'hypothèse d'un seul et même circuit de marchés de la coproduction, car comme l'affirme Romain Lecler au regard des marchés de programmes audiovisuels, il s'agit plutôt « d'une série de trajets spécifiques à chaque professionnel »" , configurés en fonction de ses intérêts et de ses relations, et caractérisé par « une grande diversité, une différentiation, et une stratification hiérarchique ", à l'instar du circuit qui englobe les festivals de cinéma, analysé par Skadi Loist ${ }^{10}$. Le nombre de projets, l'exigence qui préside à leur sélection et à celle des participants, ainsi que son intégration dans l'un des plus importants festivals au monde, place le marché berlinois en haut de la hiérarchie des marchés de la coproduction. Il entretient une relation privilégiée avec le CineMart, officialisée dans la sous-section "Rotterdam-Berlinale Express », qui accueille des projets présentés en janvier à Rotterdam, et de manière moins formalisée, des projets présents dans d'autres marchés poursuivent leur recherche de financements à Berlin en février. Inversement, des producteurs peuvent se rendre après Berlin dans d'autres marchés de la coproduction présentant une spécialisation géolinguistique afin de multiplier les rencontres déterminantes pour faire avancer le montage financier de leurs projets et élargir leurs réseaux.

Tableau 1 : Les principaux marchés de la coproduction de films

\begin{tabular}{|l|l|l|l|l|}
\hline $\begin{array}{l}\text { Marché de la } \\
\text { coproduction }\end{array}$ & Rattachement de & Date & $\begin{array}{l}\text { Année de } \\
\text { création }\end{array}$ & $\begin{array}{l}\text { pombre } \\
\text { sélectionnés } \\
\text { par an }\end{array}$ \\
\hline CineMart & $\begin{array}{l}\text { International Film Festival } \\
\text { Rotterdam, Pays-Bas }\end{array}$ & Janvier & 1984 & 16 \\
\hline $\begin{array}{l}\text { When East Meets } \\
\text { West }\end{array}$ & Trieste Film Festival, Italie & Janvier & 2010 & 21 \\
\hline
\end{tabular}




\begin{tabular}{|c|c|c|c|c|}
\hline $\begin{array}{l}\text { Berlinale Co- } \\
\text { Production } \\
\text { Market }\end{array}$ & $\begin{array}{l}\text { European Film Market, Berlin, } \\
\text { Allemagne }\end{array}$ & Février & 2004 & 35 \\
\hline $\begin{array}{ll}\text { Hong } & \text { Kong-Asia } \\
\text { Film } & \text { Financing } \\
\text { Forum } & \text { (HAF) }\end{array}$ & $\begin{array}{l}\text { Hong Kong International Film \& } \\
\text { TV Market (FILMART) }\end{array}$ & Mars & 2000 & 25 \\
\hline $\begin{array}{l}\text { Buenos Aires Lab } \\
\text { (Laboratorio de } \\
\text { produccion) }\end{array}$ & \begin{tabular}{|lrr} 
Buenos Aires & Festival \\
Internacional & de & Cine \\
Independiente & & (BAFICI), \\
Argentine & &
\end{tabular} & Avril & 2004 & 8 \\
\hline $\begin{array}{l}\text { L'Atelier de la } \\
\text { Cinéfondation }\end{array}$ & $\begin{array}{l}\text { Festival International du Film de } \\
\text { Cannes, France }\end{array}$ & Mai & 2005 & 15 \\
\hline Durban FilmMart & $\begin{array}{ll}\text { Durban } \quad \text { International } & \text { Film } \\
\text { Festival, Afrique du Sud } & \end{array}$ & Juillet & 2010 & 22 \\
\hline $\begin{array}{l}\text { CineLink Co- } \\
\text { production } \\
\text { Market }\end{array}$ & $\begin{array}{l}\text { Sarajevo Film Festival,Bosnie- } \\
\text { Herzégovine }\end{array}$ & Août & 2013 & 15 \\
\hline Open Doors Hub & $\begin{array}{l}\text { Festival international du film de } \\
\text { Locarno, Suisse }\end{array}$ & Août & 2003 & 8 \\
\hline $\begin{array}{l}\text { No Borders } \\
\text { International Co- } \\
\text { Production } \\
\text { Market }\end{array}$ & $\begin{array}{l}\text { The Independent Filmmaker } \\
\text { Project, IFP Week, New York }\end{array}$ & Septembre & 1994 & 45 \\
\hline $\begin{array}{l}\text { Europe-Latin } \\
\text { America Co- } \\
\text { production Forum }\end{array}$ & $\begin{array}{l}\text { Festival de San Sebastian, } \\
\text { Espagne }\end{array}$ & Septembre & 2012 & 17 \\
\hline $\begin{array}{ll}\text { Asian } & \text { Project } \\
\text { Market } & \end{array}$ & $\begin{array}{l}\text { Busan International Film Festival, } \\
\text { Corée du Sud }\end{array}$ & Octobre & 1998 & 28 \\
\hline $\begin{array}{l}\text { Production } \\
\text { Finance Market }\end{array}$ & $\begin{array}{l}\text { London Film Festival, Grande- } \\
\text { Bretagne }\end{array}$ & Octobre & 2007 & $\mathrm{NC}$ \\
\hline $\begin{array}{l}\text { Cinema Co- } \\
\text { Production } \\
\text { Market }\end{array}$ & $\begin{array}{l}\text { Mercato Internazionale } \\
\text { Audiovisivo, Rome, Italie }\end{array}$ & Octobre & 2006 & 20 \\
\hline $\begin{array}{l}\text { The Dubai Film } \\
\text { Connection }\end{array}$ & $\begin{array}{l}\text { Dubai International Film Festival } \\
\text { Émirats arabes unis }\end{array}$ & Décembre & 2007 & 10 \\
\hline $\begin{array}{l}\text { Le village des } \\
\text { coproductions }\end{array}$ & $\begin{array}{l}\text { Festival de Cinéma Européen des } \\
\text { Arcs, France }\end{array}$ & Décembre & 2009 & 20 \\
\hline
\end{tabular}




\section{La mise en relation des acteurs du marché de la coproduction}

Deux groupes participent au marché de la coproduction de Berlin: les producteurs des projets sélectionnés, et les financeurs potentiels, qui peuvent être des producteurs, des vendeurs internationaux, des distributeurs, des diffuseurs ou des représentants de fonds publics. L'intervention financière dans les projets se matérialise soit à travers la coproduction, soit en prenant des droits de distribution sur un territoire ou à l'international, ou à travers ces deux modalités. Le nombre de participants est passé de 285 lors de la première édition en 2004 à presque 600, originaires d'une cinquantaine de pays, en 2018. Cette progression est moins forte que celle qu'a connu le EFM ${ }^{11}$, car il y a une volonté de limiter le nombre de participants pour optimiser leur mise en relation, comme l'affirme Martina Bleis, co-directrice de la manifestation ${ }^{12}$. Les projets, précise Bleis, sont sélectionnés notamment pour « leur potentiel à intéresser des coproducteurs internationaux ", ce qui, d'après notre analyse des projets présentés entre 2013 et 2018, comporte un mélange d'intention auctoriale, universalité du sujet et ambition formelle. Par ailleurs, les demandes d'inscription des professionnels qui souhaitent rencontrer les porteurs des projets sont validées en fonction de leur expérience dans la production et la commercialisation de cinéma d'auteur transnational. Outre les demandes d'inscription qui leur sont adressées, les organisateurs du marché prennent aussi l'initiative d'inviter des professionnels susceptibles d'être intéressés par les projets sélectionnés, identifiés grâce à une veille effectuée tout au long de l'année. Chaque année sont également invitées cinq sociétés de production qui ont développé des stratégies d'internationalisation couronnées de succès, afin que leurs représentants puissent partager leur expérience avec des participants moins rompus à la pratique de la coproduction, ce qui fait de ce lieu une « zone de contact». C'est ainsi que Mary Louise Pratt désigne « des espaces sociaux où les cultures se rencontrent, se confrontent et entrent en concurrence, souvent dans des relations de pouvoir asymétriques ${ }^{13} »$. Ces espaces de rencontres favorisent la globalisation des esthétiques et des pratiques professionnelles. Malgré les différences, l'organisation ne prend en charge aucun frais lié à la participation, y compris des "invités", afin de s'assurer que tous ont des intérêts forts dans la coproduction internationale et font déjà partie du réseau plus large de la Berlinale. Pour cela, ils doivent s'acquitter d'une inscription à l'EFM, comprise entre 250 euros pour le marché seul et 475 euros si elle donne accès aussi aux projections du festival.

7 Les rendez-vous individuels constituent la colonne vertébrale du marché de la coproduction, et leur organisation révèle l'articulation des différents intérêts en jeu. La première étape consiste en la constitution d'un catalogue où figurent les 35 projets retenus, à partir d'environ 300 soumissions effectuées entre août et octobre. Pour chaque projet, le catalogue présente des informations sur l'avancement, le budget et les financements déjà acquis, un synopsis, une note d'intention, une présentation de la société de production et une biographie du réalisateur. Ces informations sont accompagnées d'indications du producteur sur l'objet de sa recherche: le montant qui reste à financer, les catégories de professionnels qu'il souhaite associer au projet, les pays préférés pour la coproduction, les concessions qu'il est prêt à faire en termes de langue, 
de lieu de tournage, de casting, d'incorporation de techniciens étrangers ou de localisation de certaines activités dans le pays coproducteur, mais aussi les contraintes existantes par rapport à ces aspects, notamment quand le projet arrive avec un autre coproducteur ou un financement public déjà associés.

8 Les professionnels inscrits reçoivent le catalogue en décembre et peuvent ensuite adresser leurs demandes de rendez-vous à l'équipe du marché, qui les transmettent aux professionnels concernés, ajoutant quelques suggestions de rencontres. Deux outils complètent la médiation nécessaire à l'organisation de la manifestation, témoignant du rôle de la technique comme élément des relations interprofessionnelles ${ }^{14}$. Le premier est la plate-forme numérique FestivalScope, qui permet le visionnage en ligne des films déjà produits ou distribués par les futurs interlocuteurs et l'accès à des informations sur la carrière des films dans le circuit de festivals. Le deuxième est le logiciel utilisé pour planifier les rendez-vous (plus de 1300 en 2018), sur la base d'un algorithme qui complète les demandes exprimées par les participants prenant en compte les attentes associées à chaque projet et la disponibilité des inscrits. Le développement de ce logiciel se poursuit depuis 2005 afin d'améliorer sa performance, intimement liée à l'efficacité et par conséquent à la notoriété du marché.

Les rendez-vous individuels se concentrent sur les deux premiers jours, le troisième étant consacré aux rencontres avec des responsables de fonds de soutien aux coproductions et des éditeurs de livres. Des représentants de seize fonds nationaux (quinze européens et un canadien) et du fonds européen Eurimages étaient présents en février 2018. De plus, une rencontre avec des éditeurs et des agents littéraires de dix livres susceptibles d'être adaptés est organisée en partenariat avec la Foire du livre de Francfort. Pendant ces trois jours, le marché de la coproduction propose en parallèle des conférences, des tables rondes et des études de cas autour de la coproduction. Ces activités reflètent une tendance de l'industrie à " faire de la recherche et à théoriser sur elle-même " ${ }^{15}$ faisant appel à des professionnels (producteurs, responsables de fonds et d'institutions ou analystes de l'industrie) qui ont un rôle majeur dans la construction d'une rhétorique et dans la transmission des valeurs, des pratiques et des connaissances qui configurent la culture professionnelle associée à la coproduction internationale de cinéma indépendant.

Ces activités ont lieu, comme celles décrites précédemment, dans le Parlement régional, contrairement à la section CoPro Series. Bien qu'intégrée au marché de la coproduction, cette section, créée en 2015, se déroule pendant le quatrième et dernier jour dans le Zoo Palast, la plus ancienne salle de cinéma du pays, détruite en 1945 et reconstruite dans les années 1950 pour accueillir des projections de la Berlinale, avant que le festival ne déplace son centre de gravité vers les nouveaux multiplexes de Potsdamer Platz ${ }^{16}$. Cette séparation spatio-temporelle marque une distance entre le secteur stratégique de la coproduction internationale de séries télévisuelles et la niche fragile et protégée, et pourtant en plein essor, de la coproduction d'un cinéma d'auteur intégré dans le « cinéma du monde ».

\section{Le marché de la coproduction et l'économie du « cinéma du monde »}

11 Nous allons maintenant examiner le rapport que dessine le marché de la coproduction de Berlin entre la pratique de la coproduction internationale et le «cinéma du monde». 
Considéré à partir des années 1960 comme altérité des cinémas occidentaux et notamment de Hollywood, son acception contemporaine prend en compte une grande diversité d'échanges globaux concernant des films et des cinéastes. Les auteurs de l'introduction à une récente compilation de textes qui examinent la notion de «cinéma du monde », constatent que les cinémas qui l'intègrent

sont définis non par une réaction clichée contre Hollywood de la part des « autres » cinémas, mais par quelque chose de beaucoup plus proche d'un réseau de défis à l'homogénéité par lequel les cinéastes et les spectateurs expérimentent, acceptent ou tentent de rejeter la mondialisation selon leurs propres termes ${ }^{17}$.

Le marché de la coproduction de Berlin soutient une internationalisation du cinéma qui incorpore les transformations d'une industrie devenue globale, remplaçant les catégories géopolitiques par une approche centrée sur des auteurs qui font partie du système industriel du cinéma indépendant ou qui sont susceptibles de l'intégrer, assimilables à la notion d'«auteur global $»^{18}$. Le cinéma produit par cette instance correspond à un segment de la production indépendante qui intègre le « cinéma du monde » et le " cinéma d'auteur » au sein de ce que Rosalind Galt et Karl Schoonover désignent comme « global art cinéma ${ }^{19}$, qui peut être traduit comme « cinéma d'auteur global ». Plus précisément, le marché de la coproduction de Berlin opère une discrimination par le budget, sélectionnant des films qui ont un coût estimé entre 1 et 4 millions d'euros ${ }^{20}$. Ce critère favorise la présence de projets et de financements européen ${ }^{21}$ et exclut tout un pan du cinéma du monde produit à moindre coût, tout comme les projets d'auteurs consacrés, qui peuvent se financer dans des contextes relationnels moins structurés. Le filtre budgétaire révèle des attentes par rapport à la qualité des films, évaluée à l'aune du potentiel de sélection en festival et d'insertion dans le circuit qui va permettre un retour sur investissement (salles d'art et essai, chaînes de télévision et plateformes de VOD).

Un total de 260 films, environ 50 pour cent des projets présentés dans le marché de la coproduction de Berlin, ont été réalisés en février 2018, la plupart dans le cadre d'une coproduction, considérée comme "le moteur qui fait avancer le marché actuellement $»^{22}$. Exceptionnellement, quelques rares projets qui ont transité par ce marché finissent par se produire avec des financements exclusivement nationaux ou incorporent des financements internationaux qui, par leur nature ou par leur montant inférieur aux seuils exigés (en général 10 pour cent du budget), ne donnent pas lieu à une coproduction officielle. Les coproductions initiées ou complétées à Berlin se caractérisent par la présence de fonds publics aux côtés de capitaux privés, apportés notamment par des diffuseurs, en particulier $\mathrm{Arte}^{23}$, et des vendeurs internationaux. Forts d'une connaissance fine de l'offre et la demande du cinéma indépendant, ainsi que de leur capacité à intervenir également en tant que coproducteurs, ces derniers contribuent de manière significative à structurer le marché global pour le "cinéma du monde ", amorcé par les fonds de soutien à la coproduction. Dans un article qui fournit des repères méthodologiques pour approcher la dimension industrielle des festivals, Marc Peranson voit les vendeurs internationaux, dont la plupart sont basés en Europe, majoritairement en France,$^{24}$ comme « les principaux acteurs de l'actuelle économie politique du festival cinématographique $»^{25}$. L'intervention en amont de ces professionnels, qui représentaient 15 pour cent des participants au marché de la coproduction de Berlin en 2018, leur permet de repérer les projets les plus intéressants et d'investir des montants plus élevés que lorsqu'ils s'occupent uniquement de l'exportation de films finalisés. 


\section{Conclusion} producteurs et les acteurs publics et privés impliqués dans l'existence d'un segment du "cinéma du monde» corrélé à la coproduction internationale. L'examen des composantes qui intègrent cette manifestation et de leurs dynamiques relationnelles fait apparaître un secteur eurocentrique et global où les acteurs partagent une culture cinématographique et un modèle d'entrepreneuriat. La culture cinématographique qui se construit et se transmet dans ce cadre est nourrie par la tradition du «cinéma du monde », redéfini à l'aune de l'émergence d'un marché global façonné par les politiques publiques, caractérisé par la centralité de l'auteur dans le financement et le marketing des films et porté par l'émergence d'un secteur dynamique des ventes internationales de films indépendants, y compris ceux qualifiés comme de niche. Le modèle économique de ce secteur repose sur des acteurs qui possèdent d'une part le goût de la découverte et de l'accompagnement d'auteurs étrangers, et d'autre part, une approche professionnelle de la production et des marchés. Les sociétés de production et de ventes internationales présentes sur le marché de la coproduction incarnent de nouvelles formes d'entrepreneuriat culturel qui combinent la prise de risque et les soutiens publics, ainsi que l'ancrage dans un contexte national et l'ouverture à des collaborations transnationales.

Dans un cadre très structuré, ce marché de la coproduction permet de compléter le financement de films, de découvrir des projets et des partenaires, et d'appréhender les codes d'une culture de la production bâtie sur l'appartenance à des réseaux internationaux. Le marché accorde par ailleurs un label de qualité aux projets et aux sociétés qui participent, facilitant ainsi leur insertion dans le circuit du financement international du cinéma d'auteur.

\section{BIBLIOGRAPHIE}

Nicolas Brigaud-Robert, « Entretien avec Fabien Lemercier », Cinéuropa, 16 mai 2017 [en ligne] http://cineuropa.org/it.aspx?t=interview\&l=fr\&did=328433\#cm (consulté le 12/01/2018).

John T. Caldwell, «Both Sides of the Fence : Blurred Distinctions in Scholarship and Production (a Portfolio of Interviews) » dans Vicky Mayer, Miranda Banks et John T. Caldwell (dir.), Production Studies: Cultural Studies of Media Industries, New York, Routledge, 2009.

Damien Charrieras, «L'apport des cultural studies à l'étude des instances de production professionnalisée de la culture ", Les Enjeux de l'information et de la communication, n 1, 2007, pp. 21-33.

Peter Cowie, The Berlinale. The Festival, Berlin, Bertz + Fischer, 2010. 
Thomas Elsaesser, « The Global Author: Control, Creative Constraints and Performative Self Contradiction » dans Seung-hoon Jeong et Jeremi Szaniewski (dir.), The Global Auteur. The Politics of Authorship in $21^{\text {st }}$ Century Cinema, New York, Bloomsbury, 2016.

Marijke De Valck, «Supporting art cinema at a time of commercialization: Principles and Practices, the Case of the International Film Festival Rotterdam », Poetics, vol. 42, 2014, pp 40-59.

Rosalind Galt, et Karl Schoonover (dir.), Global Art Cinema. New Theories and Histories, New York, Oxford University Press, 2010.

Janet Harbord, Film Cultures, London, Sage, 2002.

Dina Iordanova, « The Film Festival as an Industry Node », Media Industries Journal vol. 1, $\mathrm{n}^{\circ} 3$, 2015 , pp. 7-11. [en ligne] DOI : http://dx.doi.org/10.3998/mij.15031809.0001.302 (consulté le 6/01/2018).

Bruno Latour, Changer la société, refaire de la sociologie, Paris, La Découverte, 2006.

Romain LECLER, «La montée des marchés à Cannes. Le circuit des échanges audiovisuels internationaux, sa chronologie et ses trajets professionnels ", Réseaux, vol. 200, n 6, 2016, pp. 209-242.

Sidney Levine, « The Berlinale and EFM from my POV » Indiwire, 26/02/2014 [en ligne] http:// www.indiewire.com/2014/02/the-berlinale-and-efm-from-my-pov-168899/ (consulté le 10/01/2018).

Skadi Loist, « The film festival circuit : networks, hierarchies, and circulation » dans Film Festivals : History, Theory, Method, Practice, Marijke de Valck et al (dir.) London et New York, Routledge, 2016.

Geoffrey Macnab, « The match-makers - new co-production markets ", Screen Daily, 12/10/2007, [en ligne] https://www.screendaily.com/the-match-makers-new-co-productionmarkets/4035208.article (consulté le 12/02/2018)

Mark Peranson, « First You Get the Power, Then You Get the Money: Two Models of Film Festivals. Cineaste, vol. 33, n 3, 2008, pp. 37-43.

Mary Louise Pratt, « Arts of the Contact Zone », Profession n 91, New York, Modern Language Association, 1991, pp. 33-40.

Ragan Rhyne, «Film festival circuits and stakeholders » dans Film Festival Yearbook 1: The Festival Circuit, Dina Iordanova et Ragan Rhyne (dir.), St. Andrews, St Andrews Film Studies 2009, pp. 9-22. Rob Stone, Paul Cooke, Stephanie Dennison et Alex Marlow-Mann, The Routledge Companion to World Cinema, New York, Routledge, 2018.

\section{NOTES}

1. Damien Charrieras, «L'apport des cultural studies à l'étude des instances de production professionnalisée de la culture ", Les Enjeux de l'information et de la communication, n 1, 2007, p. 6. 2. Le site du Film Festival Research Network présente une bibliographie annotée relative à ce domaine, établie par Marijke de Valck et Skadi Loist, fondatrices du réseau. Elle est consultable sur http://www.filmfestivalresearch.org/index.php/ffrn-bibliography/

3. Ragan Rhyne, "Film festival circuits and stakeholders" dans Film Festival Yearbook 1: The Festival Circuit, Dina Iordanova et Ragan Rhyne (dir.), St. Andrews, St Andrews Film Studies, 2009, pp. 9-22. 
4. Marijke De Valk, «Supporting art cinema at a time of commercialization: Principles and Practices, the Case of the International Film Festival Rotterdam », Poetics 42, n 1, 2014, pp 40-59.

5. Dina Iordanova, «The Film Festival as an Industry Node», Media Industries Journal, vol. 1, n³, 2015. [en ligne] DOI :http://dx.doi.org/10.3998/mij.15031809.0001.302

6. Selon Janet Harbord, Film Cultures, London, Sage, 2002, p.42.

7. Par exemple, Trois jours à Quiberon (Emily Atef, Allemagne/France, 2018), Soy Nero (Rafi Pitts, Allemagne/France/Mexique, 2016) ou Une femme fantastique (Sebastián Lelio, Chili/Allemagne / États-Unis/Espagne, 2017), pour ne citer que quelques films récents projetés dans la compétition officielle.

8. Selon les chiffres communiqués sur le site du programme MEDIA : https://eacea.ec.europa.eu/ sites/eacea-site/files/list_of_selected_projects_6.pdf, consulté le 06/08/2018.

9. Romain LECLER, «La montée des marchés à Cannes. Le circuit des échanges audiovisuels internationaux, sa chronologie et ses trajets professionnels ", Réseaux, vol. 200, n 6, 2016, p.15

10. Skadi Loist, "The film festival circuit: networks, hierarchies, and circulation " dans Film Festivals: History, Theory, Method, Practice, Marijke de Valck, Brendan Kredell, et Skadi Loist (dir.), Londres et New York, Routledge, 2016, p.50.

11. Le EFM est passé sur la même période de 3.500 à 10.000 participants, dont 1600 acheteurs (distributeurs, diffuseurs et représentants de plateformes numériques).

12. Entretien réalisé le 18/02/2018 à Berlin.

13. Mary Louise Pratt M, "Arts of the Contact Zone». Profession $n^{\circ} 91$. New York, Modern Language Association, 1991, p.33.

14. Ce rôle, et plus généralement l'incorporation de la technique à l'acception du social, a été mis en lumière par la sociologie de l'acteur-réseau. Voir Bruno Latour, Changer la société, refaire de la sociologie, Paris, La Découverte, 2006, pp. 101-106.

15. John T. Caldwell «Both Sides of the Fence: Blurred Distinctions in Scholarship and Production (a Portfolio of Interviews) » dans Vicky Mayer, Miranda Banks et John T. Caldwell (dir.), Production Studies: Cultural Studies of Media Industries, New York, Routledge, 2009, p. 215.

16. Peter Cowie retrace l'histoire du Festival et ses transformations dans The Berlinale. The Festival . Berlin, Bertz + Fischer, 2010.

17. Rob Stone, Paul Cooke, Stephanie Dennison, Alex Marlow-Mann, The Routledge Companion to World Cinema, New York, Routledge, 2018, p.3. «Are defined not by any clichéd reaction against Hollywood by "other" cinemas but by something much closer to a network of challenges to homogeneity by which filmmakers and film-watchers experience, accept, or attempt to reject globalisation on their own terms.»

18. Voir Thomas Elsaesser, «The Global Author: Control, Creative Constraints and Performative Self Contradiction » dans Seung-hoon Jeong et Jeremi Szaniewski (dir.), The Global Auteur. The Politics of Authorship in 21 ${ }^{\text {st }}$ Century Cinema, New York, Bloomsbury, 2016, p. 38.

19. Ces auteurs réfutent la logique binaire selon laquelle le cinéma d'auteur a été assimilé à une vision étroite et réactionnaire de l'international, plutôt qu'à des cadres ouverts, radicaux ou controversés, comme ceux qui offrent le world cinema, le post-colonialisme ou la globalisation. Ils envisagent, au contraire, le cinéma d'auteur comme la catégorie la mieux placée pour aborder l'émergence d'une culture mondiale et l'influence des financements internationaux dans les formes filmiques. Voir Rosalind Galt, Karl Schoonover, «Introduction: The Impurity of Art Cinema » dans Rosalind Galt, Karl Schoonover (dir.), Global Art Cinema. New Theories and Histories, New York, Oxford University Press, 2010, pp. 9-10.

20. Même si le plafond indiqué est de 20 millions d'euros, le seuil de 4 millions a été rarement dépassé.

21. Environ 75 pour cent des participants sont européens, le reste venant principalement du Canada, des États-Unis et d'Amérique Latine. Concernant les projets, un peu plus de la moitié sont issus d'un pays européen. 
22. Selon Sidney Levine, dans "The Berlinale and EFM from my POV » Indiwire, 26/02/2014 [en ligne] http://www.indiewire.com/2014/02/the-berlinale-and-efm-from-my-pov-168899/ (consulté le 10/01/2018).

23. Le diffuseur franco-allemand décerne par ailleurs chaque année le « Prix Arte International », d'un montant de 6000 euros, à un projet présent à cette manifestation.

24. Nicolas Brigaud-Robert, co-fondateur de la société Playtime, estime dans un entretien publié dans Cinéuropa en 2017 que 70 pour cent des sociétés européennes de ventes internationales de films sont françaises.

25. Mark Peranson, «First You Get the Power, Then You Get the Money: Two Models of Film Festivals ». Cineaste, vol. 33, n³, 2008, p.29.

\section{RÉSUMÉS}

Cet article vise à identifier l'organisation et les enjeux d'un marché de la coproduction, avec une approche qui introduit des problématiques industrielles et économiques dans l'étude d'un espace de production de cinéma du monde. Prenant comme exemple le marché de la coproduction de la Berlinale, conçu pour faciliter la rencontre entre des producteurs porteurs d'un projet de film et de potentiels financeurs internationaux, il s'attache à appréhender les dynamiques relationnelles entre les parties impliquées et la culture cinématographique qui se forge et se transmet au sein de cette manifestation.

This article aims to identify the organization and the stakes of a co-production market, with an approach that introduces industrial and economic issues into the study of a production space of world cinema. Taking as an example the Berlinale's co-production market, designed to connect producers with a film project and potential international financers, it endeavors to explain the relational dynamics between the participants and the cinematographic culture that is forged and transmitted within this event.

\section{AUTEUR}

\section{ANA VINUELA}

Maîtresse de conférences à l'Université Paris Diderot-Paris 7 et membre du laboratoire CERILAC (Centre d'Études et de Recherches Interdisciplinaires en Lettres, Arts et Cinéma). Elle enseigne la socio-économie du cinéma et de l'audiovisuel et la production. Docteur en Sciences de l'information et de la communication de l'Université Complutense de Madrid, ses recherches portent sur le dialogue entre la France et le « cinéma du monde », les industries cinématographiques et audiovisuelles et les politiques publiques dans ces secteurs. 